\title{
REAL PLANE ALGEBRAIC CURVES WITH ASYMPTOTICALLY MAXIMAL NUMBER OF EVEN OVALS
}

\author{
Erwan Brugallé
}

\begin{abstract}
It is known for a long time that a nonsingular real algebraic curve of degree $2 k$ in the projective plane cannot have more than $\frac{7 k^{2}}{2}-\frac{9 k}{4}+\frac{3}{2}$ even ovals. We show here that this upper bound is asymptotically sharp, that is to say we construct a family of curves of degree $2 k$ such that $\frac{p}{k^{2}} \rightarrow_{k \rightarrow \infty} \frac{7}{4}$, where $p$ is the number of even ovals of the curves. We also show that the same kind of result is valid dealing with odd ovals.
\end{abstract}

\section{Introduction}

The set $\mathbb{R} A$ of the real points of a nonsingular real algebraic curve in $\mathbb{R} P^{2}$ is a disjoint union of circles. Each of these circles either disconnects $\mathbb{R} P^{2}$ or not. In the former case, such a circle is called an oval of $\mathbb{R} A$. The part of $\mathbb{R} P^{2}$ cut along this oval which is homeomorphic to a disk is called the interior of the oval. An oval of a curve which contains no other ovals of the curve is called an empty oval. All the connected components of a curve of even degree are ovals. For such a curve, the ovals which are contained in an even (resp. odd) number of ovals are called even (resp. odd) ovals. Given a real plane algebraic curve of degree $2 k$, its number of even (resp. odd) ovals will be denoted by $p$ (resp. $n$ ). This separation of ovals in two groups is important for many reasons. One of them is the fact that curves with many even ovals can be used to construct real algebraic surfaces with big Betti numbers (see section [6).

What are the maximal possible values for $p$ and $n$ with respect to $k$ ? The first step in the study of this problem is due to V. Ragsdale. In 1906, she conjectured in Rag06 that $p \leq \frac{3 k(k-1)}{2}+1$ and $n \leq \frac{3 k(k-1)}{2}$. About 30 years later, I. G. Petrovsky proved in Pet33 that $p-n \leq \frac{3 k(k-1)}{2}+1$ and $n-p \leq \frac{3 k(k-1)}{2}$ (these inequalities were also conjectured by Ragsdale), and formulated a conjecture similar to Ragsdale's one (it seems clear that Petrovsky was not familiar with Ragsdale's work). Combining the first Petrovsky inequality with the Harnack Theorem (which gives an upper bound for the number of connected components of a real plane algebraic curve with respect to its degree) one can obtain the following upper bounds for $p$ and $n$ :

$$
p \leq \frac{7 k^{2}}{4}-\frac{9 k}{4}+\frac{3}{2} \text { and } n \leq \frac{7 k^{2}}{4}-\frac{9 k}{4}+1
$$

The first counterexamples to Ragsdale's conjecture for $n$ (but not to Petrovsky's one) were constructed by O. Ya. Viro in the late 70's (see Vir89]). In 1993, I. Itenberg gave in [te93] counterexamples to Ragsdale's and Petrovsky's conjectures. He has constructed for every positive integer $k$ curves of degree $2 k$ with $\frac{13 k^{2}}{8}+O(k)$ even ovals and curves of degree $2 k$ with $\frac{13 k^{2}}{8}+O(k)$ odd ovals. These lower bounds were successively improved by B. Haas (see Haa95) and Itenberg (see [te01]). The best lower bound known before the present paper was $\frac{81 k^{2}}{48}+O(k)$ for both $p$ and $n$. We point out the fact that that no counterexamples of Ragsdale's conjectures is known among curves with the maximal number of connected components. 
All these constructions are based on a particular case of the so called Viro method (see [Vir84, [Vir]), the combinatorial patchworking. One can note that dealing with non convex triangulations (and so with pseudo-holomorphic curves, see [S02]), F. Santos ( $\underline{\underline{\operatorname{San}}})$ constructed curves with $\frac{17 k^{2}}{10}+O\left(k^{\frac{3}{2}}\right)$ even ovals.

It seemed to us that the $T$-construction is more or less "rigid" and that the general Viro method gives one more flexibility and possibilities to construct real algebraic curves. Then, we resumed the work of Itenberg and Santos in this way, trying to increase the density of even ovals. It turned out that gluing curves whose Newton polygon is not anymore a triangle but an hexagon, it was possible to prove that the upper bounds given by the Harnack theorem and the Petrovsky inequalities are asymptotically sharp.

This is the main result of this article.

Theorem 1.1 There exists a family $\left(C_{2 k}\right)_{k \geq 0}$ of nonsingular real algebraic curves of degree $2 k$ in $\mathbb{R} P^{2}$ such that

$$
\lim _{k \rightarrow \infty} \frac{p}{k^{2}}=\frac{7}{4}
$$

There exists a family $\left(C_{2 k}\right)_{k \geq 0}$ of nonsingular real algebraic curves of degree $2 k$ in $\mathbb{R} P^{2}$ such that

$$
\lim _{k \rightarrow \infty} \frac{p}{k^{2}}=\frac{7}{4}
$$

Proof. The assertion relative to $p$ is a direct consequence of corollary 3.5. The assertion relative to $n$ can be proved, as in Ite93 and Ite01, by a small modification of the construction given in section 3 (see Figure 6).

This article is organized as follows : in section 2 we recall some facts about rational geometrically ruled surfaces. In section 3 we prove the first part of Theorem 1.1. The constructions in this section are based on the classical Viro method. We assume in this section the existence of some special curves in rational geometrically ruled surfaces. The construction of the latter curves are based on the less classical real rational graphs theoretical method. Section 4 is devoted to the definition and properties of such graphs, and in section [5] we construct the special curves used in section [3 In section [6. we give some applications of Theorem 1.1 to real algebraic surfaces.

Acknowledgment. I am grateful to Ilia Itenberg for useful discussions and advisements and to Olivier Le Gal for his art in hexagons counting.

\section{Rational geometrically ruled surfaces}

The $n^{\text {th }}$ rational geometrically ruled surface, denoted by $\Sigma_{n}$, is the surface obtained by taking four copies of $\mathbb{C}^{2}$ with coordinates $(x, y),\left(x_{2}, y_{2}\right),\left(x_{3}, y_{3}\right)$ and $\left(x_{4}, y_{4}\right)$, and by gluing them along $\left(\mathbb{C}^{*}\right)^{2}$ with the identifications $\left(x_{2}, y_{2}\right)=\left(1 / x, y / x^{n}\right),\left(x_{3}, y_{3}\right)=(x, 1 / y)$ and $\left(x_{4}, y_{4}\right)=\left(1 / x, x^{n} / y\right)$. Let us denote by $E$ (resp. $B$ and $F$ ) the algebraic curve in $\Sigma_{n}$ defined by the equation $\left\{y_{3}=0\right\}$ (resp. $\{y=0\}$ and $\{x=0\})$. The coordinate system $(x, y)$ is called standard. The projection $\pi:(x, y) \mapsto x$ on $\Sigma_{n}$ defines a $\mathbb{C} P^{1}$-bundle over $\mathbb{C} P^{1}$. We have $B \circ B=n, F \circ F=0$ and $B \circ F=1$. The surface $\Sigma_{n}$ has a natural real structure induced by the complex conjugation in $\mathbb{C}^{2}$, and the real part of $\Sigma_{n}$ is a torus if $n$ is even and a Klein bottle if $n$ is odd. The restriction of $\pi$ on $\mathbb{R} \Sigma_{n}$ defines a pencil of lines denoted by $\mathcal{L}$.

The group $H_{2}\left(\Sigma_{n}, \mathbb{Z}\right)$ is isomorphic to $\mathbb{Z} \times \mathbb{Z}$ and is generated by the classes of $B$ and $F$. Moreover, one has $E=B-n F$. An algebraic curve on $\Sigma_{n}$ is said to be of bidegree $(k, l)$ if it realizes 
the homology class $k B+l F$ in $H_{2}\left(\Sigma_{n}, \mathbb{Z}\right)$. A curve of bidegree $(3,0)$ is called a trigonal curve on $\Sigma_{n}$.

In the rational geometrically ruled surfaces, we study real curves up to isotopy with respect to $\mathcal{L}$. Two curves are said to be isotopic with respect to the fibration $\mathcal{L}$ if there exists an isotopy of $\Sigma_{n}$ which brings the first curve to the second one, and which maps each line of $\mathcal{L}$ in another line of $\mathcal{L}$. In this paper, curves in a rational geometrically ruled surface are depicted up to isotopy with respect to $\mathcal{L}$.

\section{Construction of real algebraic curves with many even ovals}

We will use the Viro method to construct real plane algebraic curves. The unfamiliar readers can refer to Vir84, and Vir].

In this section, we will use the following proposition which will be proved in section 5 .

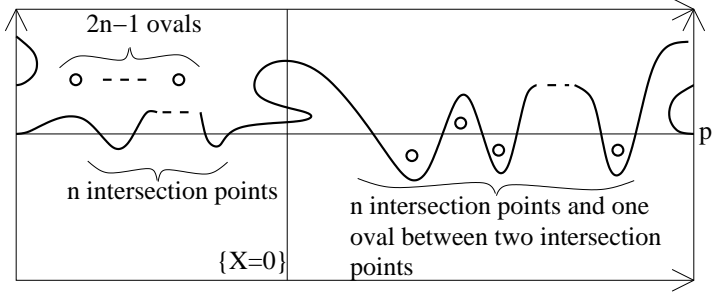

a)

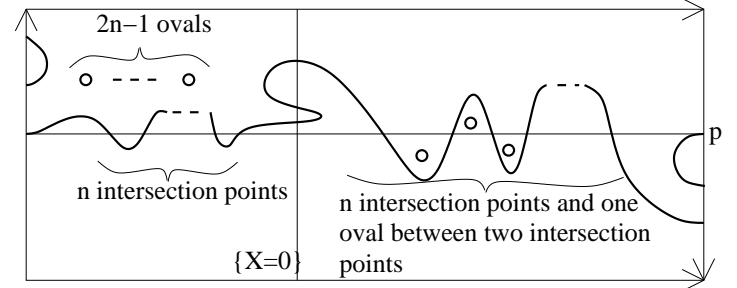

b)

Figure 1:

Proposition 3.1 For any $n \geq 1$, there exists a maximal real algebraic trigonal curve $C_{n}$ in $\Sigma_{n}$ realizing the $\mathcal{L}$ - scheme and whose position with respect to the axis $\{Y=0\}$ and $\{X=0\}$ is depicted in Figure [1 a) if $n$ is even and [1b) if $n$ is odd, where $p$ is a tangency point of order $n$ of $C_{n}$ and the axis $\{Y=0\}$.

For example, the curve for $C_{4}$ is depicted on figure 2

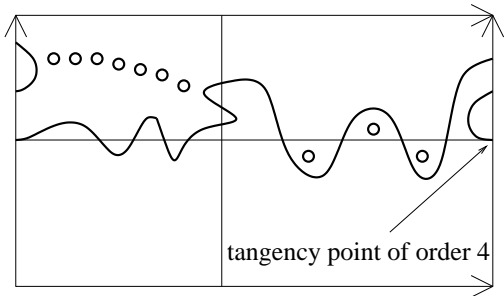

a)

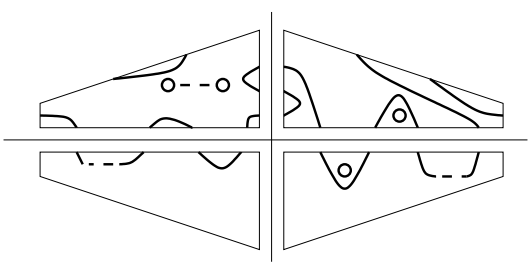

b)

Figure 2:

Let us fix an even integer $n$.

The Newton polygon of the curve $C_{n}$ is the quadrangle with vertices $(0,0),(2 n, 0),(2 n, 1)$ and $(0,3)$ and the chart of $C_{n}$ is depicted on Figure 2b) (we disjointed the 4 symmetric copies of the Newton polygon of $C_{n}$ for convenience). Moreover, performing the transformation $\widetilde{Y}=\lambda Y$ if necessary, we can assume that the truncation of $C_{n}$ on $[(0,3) ;(2 n, 1)]$ is $\alpha Y^{3}+\beta Y^{2} X^{n}+\alpha Y X^{2 n}$ with $\alpha$ and $\beta$ 
two real numbers. Let us denote by $H_{n}$ the hexagon obtained by gluing the charts of 4 birational transforms of $C_{n}$ as depicted on Figure 3 a).

Let us fix an integer $k$ and denote by $T_{2 k}$ the triangle with vertices $(0,0),(2 k, 0)$ and $(0,2 k)$. We start a subdivision of $T_{2 k}$ in the following way : for each integers $l$ and $h$, we put the hexagon $H_{n}$ centered in the point $(1+2 n+4 l, 3+8 h)$ or $(1+4 n+4 l, 7+8 h)$ if this hexagon is contained in $T_{2 k}$. In this way, we obtain the beginning of a patchwork of a real plane curve of degree $2 k$ as depicted on Figure 4 (here were chose $\mathrm{n}=4$ for convenience).

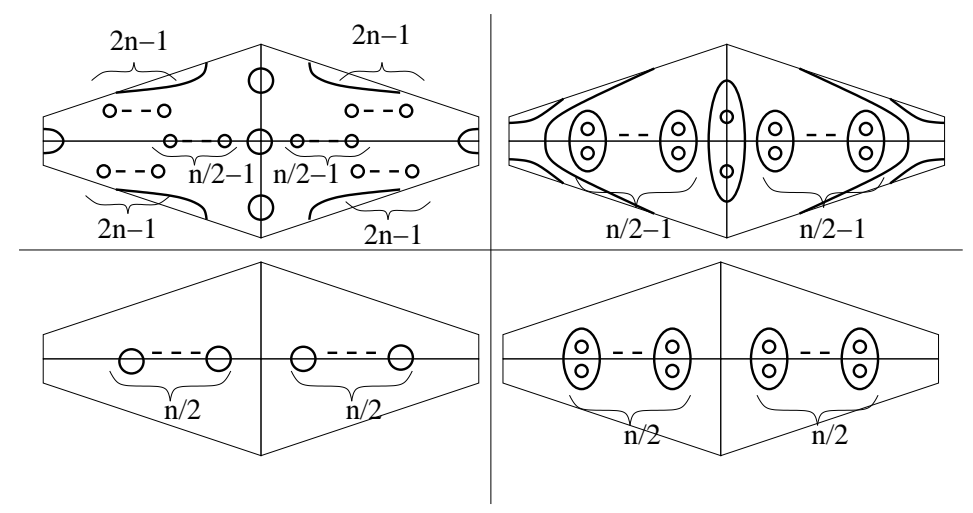

a)

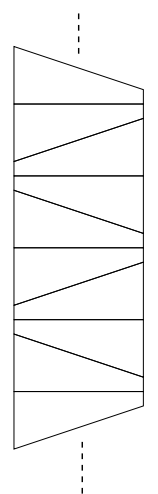

b)

Figure 3:

Lemma 3.2 The union of all the hexagons can be a part of a convex subdivision of $T_{2 k}$.

Proof. The union of the hexagons can be decomposed in vertical strips as depicted on Figure $3 \mathrm{~b}$ ). Given any convex function on the left edge of the strip, one can extend it to a convex function on the whole strip which induces this subdivision.

Suppose we are given an extension of our beginning of patchwork to the whole $T_{2 k}$, satisfying the hypothesis of the Viro Theorem (see Vir84]). Then, by Viro's Theorem, we obtain a real algebraic curve of degree $2 k$ in $\mathbb{R} P^{2}$, which we will denote by $C_{k}^{n}$. Next, let us choose such an extension such that (see Figure 5) :

- each oval of $C_{k}^{n}$ lying in the half plane $\{x<0\}$ and coming from an hexagon is even and not contained in another oval of the curve,

- each oval of $C_{k}^{n}$ lying in the quadrant $\{x>0\}$ and coming from an empty oval of an hexagon is even and contained in two other ovals of the curve,

where the coordinates system is the one given by the chart of $C_{k}^{n}$.

It is clear that such an extension exists. One can construct curves we need to complete our patchwork by the classical small perturbation method (see, for example, Vir89]). The convexity condition can be ensured, for example, by keeping on decomposing $T_{2 k}$ in strips.

Lemma 3.3 Each hexagon contributes of at least $14 n-5$ even ovals to the curve $C_{k}^{n}$.

Proof. Straightforward.

Lemma 3.4 The curve $C_{k}^{n}$ has at least $\frac{7 k^{2}}{4}-\frac{5 k^{2}}{8 n}-21 n k+\frac{15 k}{2}+63 n^{2}-\frac{45 n}{2}$ even ovals. 


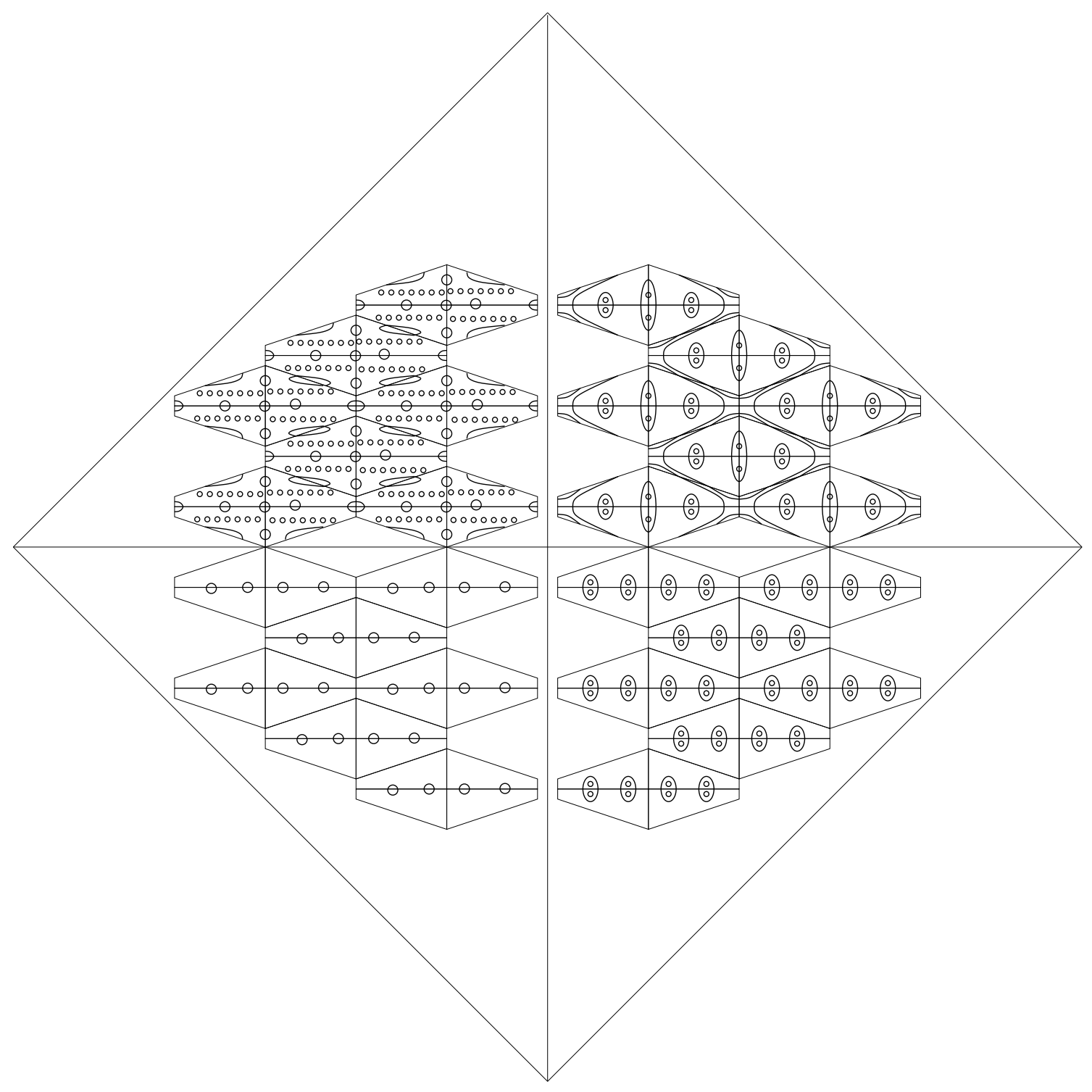

Figure 4:

Proof. According to Lemma 3.3 each hexagon $H_{n}$ in the patchwork of the curve $C_{k}^{n}$ gives at least $14 n-5$ even ovals. Then, if the patchwork contains $N$ hexagons $H_{n}$, the curve $C_{k}^{n}$ will have at least $N(14 n-5)$ even ovals. The triangle $T_{2 k}^{\prime}$ with vertices $(6 n, 6 n),(2 k-12 n, 6 n)$ and $(6 n, 2 k-12 n)$ is contained in the union of the hexagons, so

$$
N \geq \frac{\operatorname{Area}\left(T_{2 k}^{\prime}\right)}{\operatorname{Area}\left(H_{n}\right)}=\frac{(k-6 n)^{2}}{8 n} .
$$

Hence the number of even ovals of $C_{k}^{n}$ is at least $\frac{(k-6 n)^{2}}{8 n}(14 n-5)$. Developing this quantity, we obtain the lower bound stated in the lemma.

The same construction can be done with an odd integer $n$. The curve obtained is also denoted by $C_{k}^{n}$ and the lower bound of lemma 3.4 for its number of even oval is still valid.

Now we are able to prove the main theorem of this paper. We denote the integer part of a real $r$ by $[r]$. 


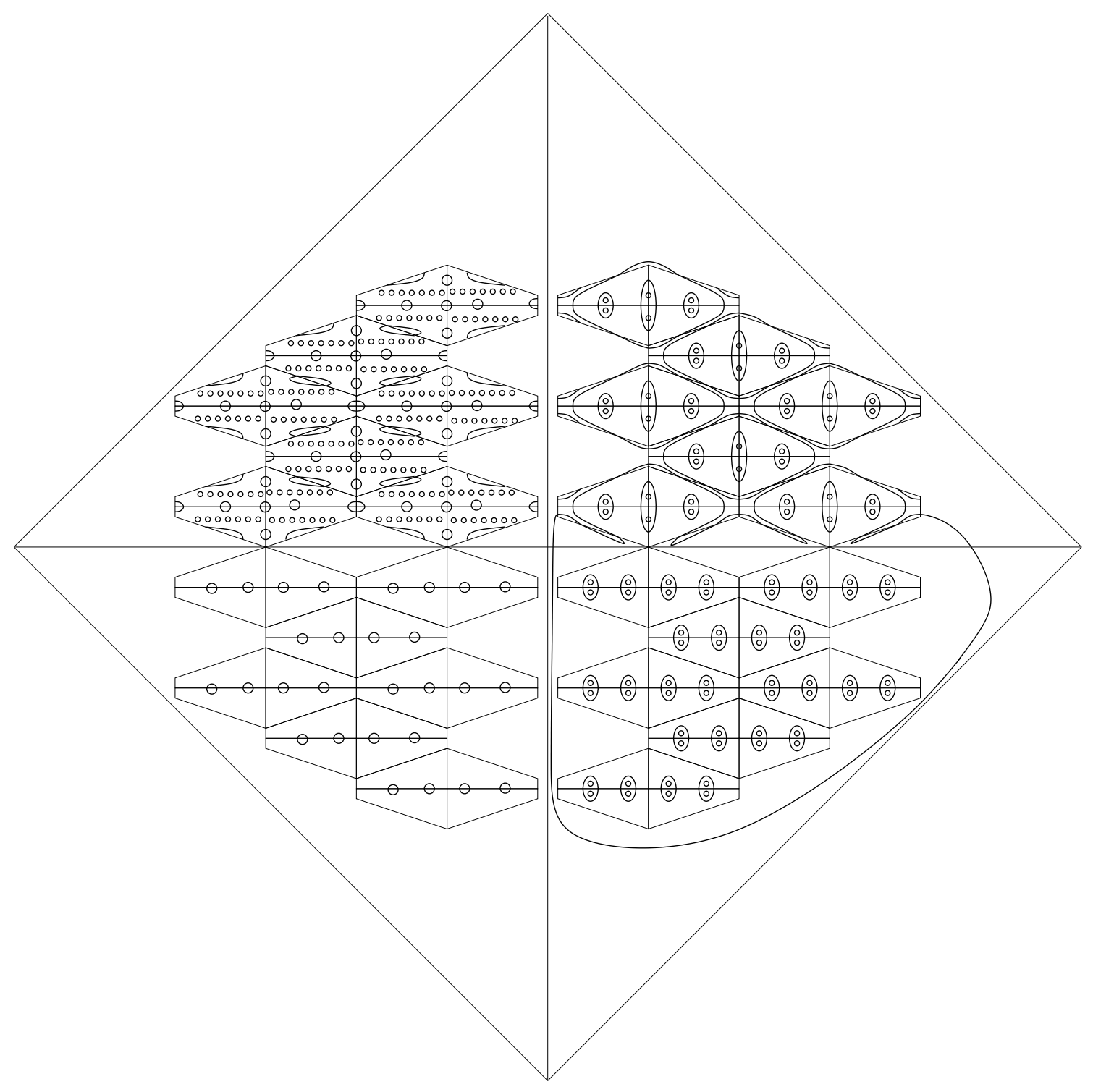

Figure 5:

Corollary 3.5 The curve $C_{k}^{[\sqrt{k}]}$ has $\frac{7 k^{2}}{4}+O\left(k^{\frac{3}{2}}\right)$ even ovals.

\section{Real rational graphs on $\mathbb{C} P^{1}$}

This section deals with the following problem : given a real arrangement of roots of three real polynomials (called a root scheme below), does there exist two real polynomials $P$ and $Q$ such that the real roots of $P, Q$ and $P+Q$ realize the given arrangement?

This question can be reformulated in terms of existence of a certain graph on $\mathbb{C} P^{1}$ (called a real rational graph below).

We start with the following fact : to any rational map $f: \mathbb{C} P^{1} \rightarrow \mathbb{C} P^{1}$, one can associate a natural graph on $\mathbb{C} P^{1}$, namely $f^{-1}\left(\mathbb{R} P^{1}\right)$. This correspondence is used for example by S. Natanzon, B. Shapiro and A. Vainshtein to classify topologically generic real rational maps (see NSV02 and 


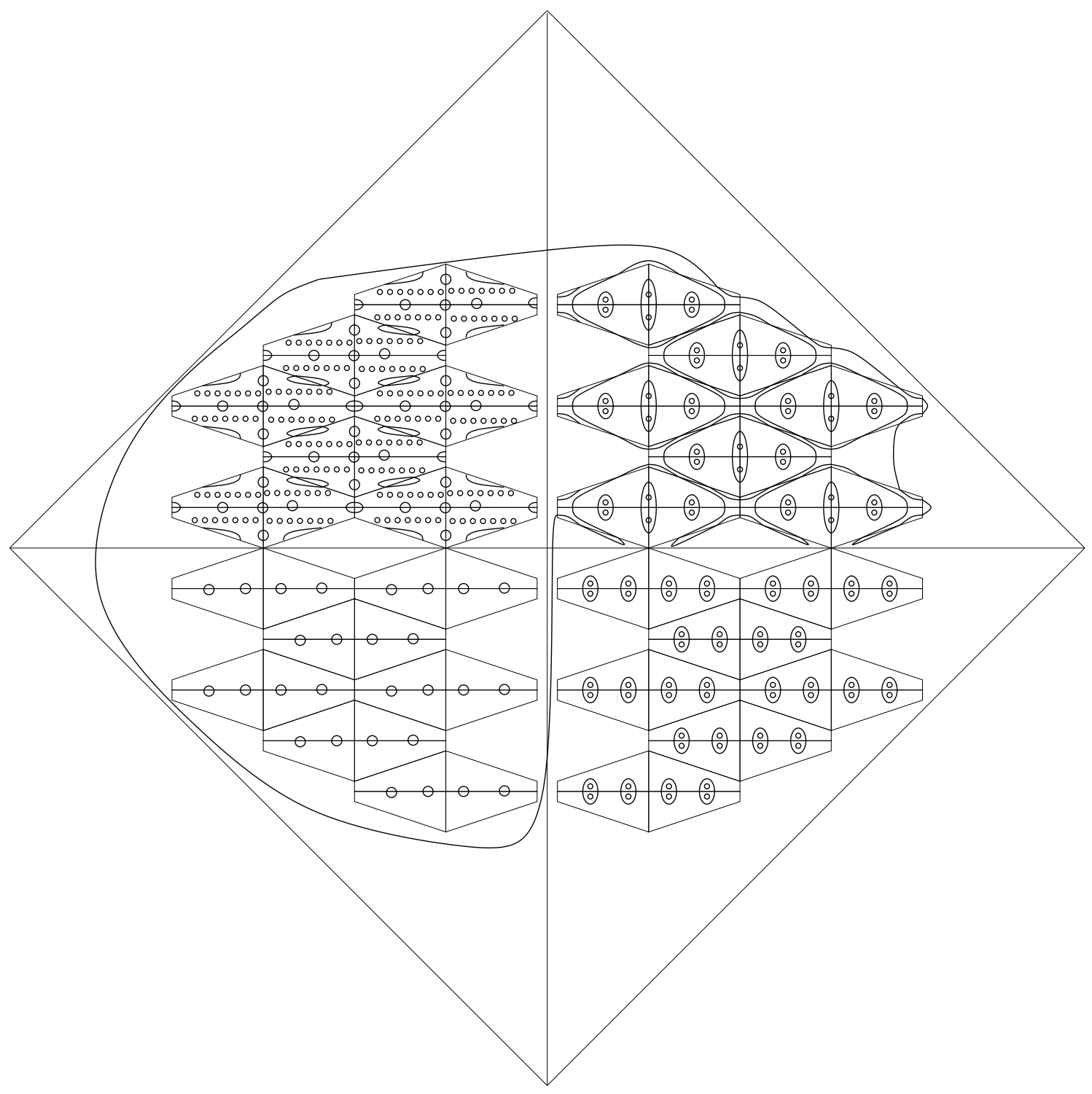

Figure 6:

[SV03]). An other application of these graphs has been exploited by A. Zvonkin ([Zvo]). He used these graphs to study the minimal degree of $P^{3}-Q^{2}$, where $P$ and $Q$ are complex polynomials of degrees $2 k$ and $3 k$, respectively. Following Zvonkin, Orevkov proposed in Ore03 a new way to construct real algebraic trigonal curves in rational geometrically ruled surfaces. Using Orevkov's approach, we proved in $\mathrm{Bru}$, the non-realizability of some $\mathcal{L}$-schemes by real algebraic trigonal curves. All the graphs considered in both these papers are special cases of real rational graphs, called real trigonal graphs in $\mathrm{Bru}$. However, arguments used in Ore03 are valid in the more general context of real rational graphs.

Definition 4.1 A root scheme is a k-uplet $\left(\left(l_{1}, m_{1}\right), \ldots,\left(l_{k}, m_{k}\right)\right) \in(\{p, q, r\} \times \mathbb{N})^{k}$ with $k$ a natural number (here, $p, q$ and $r$ are symbols and do not stand for natural numbers).

A root scheme $\left(\left(l_{1}, m_{1}\right), \ldots,\left(l_{k}, m_{k}\right)\right)$ is realizable by polynomials of degree $n$ if there exist two real polynomials in one variable of degree $n$, with no common roots, $P(X)$ and $Q(X)$ such that if $x_{1}<$ 
$x_{2}<\ldots<x_{k}$ are the real roots of $P, Q$ and $P+Q$, then $l_{i}=p$ (resp., $\left.q, r\right)$ if $x_{i}$ is a root of $P$ (resp., $Q, P+Q$ ) and $m_{i}$ is the multiplicity of $x_{i}$.

The polynomials $P, Q$ and $P+Q$ are said to realize the root scheme $\left(\left(l_{1}, m_{1}\right), \ldots,\left(l_{k}, m_{k}\right)\right)$.

In a root scheme, we will abbreviate a sequence $S$ repeated $u$ times by $S^{u}$.

From now on, let $R S$ be a root scheme and suppose that $R S$ is realized by $P, Q$ and $P+Q$ of degree $n$. Put $R(X)=P(X)+Q(X)$ and consider the rational function $f(X)=\frac{R(X)}{Q(X)}=\frac{P(X)}{Q(X)}+1$. Color and orient $\mathbb{R} P^{1}$ as depicted in Figure $\left.7 \mathrm{a}\right)$. Let $\Gamma$ be $f^{-1}\left(\mathbb{R} P^{1}\right)$ with the coloring and the orientation induced by those chosen on $\mathbb{R} P^{1}$. Then, $\Gamma$ is a colored and oriented graph on $\mathbb{C} P^{1}$, invariant under the action of the complex conjugation. The colored and oriented graph on $\mathbb{R} P^{1}$ obtained as the intersection of $\Gamma$ and $\mathbb{R} P^{1}$ can clearly be extracted from $R S$.

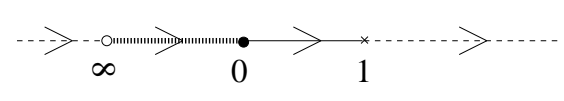

a)

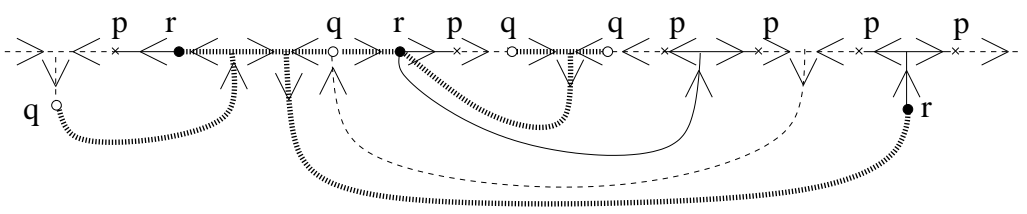

b)

Figure 7:

Definition 4.2 The colored and oriented graph on $\mathbb{R} P^{1}$ constructed above is called the real graph associated to $R S$.

Definition 4.3 Let $\Gamma$ be a graph on $\mathbb{C} P^{1}$ invariant under the action of the complex conjugation and $\pi: \Gamma \rightarrow \mathbb{R} P^{1}$ a continuous map. Then the coloring and orientation of $\mathbb{R} P^{1}$ shown in Figure $7 a$ ) defines a coloring and an orientation of $\Gamma$ via $\pi$.

The graph $\Gamma$ equipped with this coloring and this orientation is called a real rational graph if

- any vertex of $\Gamma$ has an even valence,

- any connected component $D$ of $\mathbb{C} P^{1} \backslash \Gamma$ is homeomorphic to an open disk,

- for any connected component $D$ of $\mathbb{C} P^{1} \backslash \Gamma$, one has $\pi_{\mid \partial D}$ is a covering of $\mathbb{R} P^{1}$ of degree $d_{D}$.

The sum of the degrees $d_{D}$ for all connected component $D$ of $\{\operatorname{Im}(z)>0\} \backslash \mathbb{R} P^{1}$ of is called the degree of $\Gamma$.

The importance of real rational graphs is given by the following proposition.

Proposition 4.4 Let $R S$ be a root scheme and $G$ its real graph. Then $R S$ is realizable by polynomials of degree $n$ if and only if there exists a real rational graph $\Gamma$ of degree $n$ such that $\Gamma \cap \mathbb{R} P^{1}=G$.

Proof. The proof used in Ore03] to construct real trigonal curves in $\Sigma_{n}$ can be used here in the same way.

For example, the root scheme $((p, 1),(r, 1),(q, 2),(r, 3),(p, 1),(q, 1),(q, 1),(p, 1),(p, 1),(p, 1),(p, 1))$ is realizable by polynomials of degree 6 as it is depicted on Figure $7 \mathrm{~b})$.

Lemma 4.5 Let $R S=\left(\left(l_{1}, m_{1}\right), \ldots,\left(l_{k}, m_{k}\right)\right)$ a root scheme such that there exist $i$ and $s$ such that $\forall j \in\{i, \ldots, i+s\}, l_{j}=l_{i}$. Define the root scheme $R S^{\prime}=\left(\left(l_{1}^{\prime}, m_{1}^{\prime}\right), \ldots,\left(l_{k-s}^{\prime}, m_{k-s}^{\prime}\right)\right)$ by 
- $\left(l_{t}^{\prime}, m_{t}^{\prime}\right)=\left(l_{t}, m_{t}\right)$ for $t<i$,

- $\left(l_{i}^{\prime}, m_{i}^{\prime}\right)=\left(l_{i}, m_{i}+\ldots+m_{i+s}\right)$,

- $\left(l_{t}^{\prime}, m_{t}^{\prime}\right)=\left(l_{t-s}, m_{t-s}\right)$ for $t>i+s$,

Then $R S$ is realizable by polynomials of degree $n$ if and only if $R S^{\prime}$ is realizable by polynomials of degree $n$.

Proof. Straightforward.

\section{Construction of reducible curves with a deep tangency point}

Let us define the root schemes $R S_{n}$ by

- $\left((p, n),\left[(r, 1),(q, 1)^{2},(r, 1)\right]^{k},(r, 1),(q, 1),(p, 1),(r, 1),\left[(p, 1),(r, 1)^{2},(p, 1)\right]^{k},(q, 1)^{n}\right)$ if $n=2 k+$ 1 ,

- $\left((p, n),\left[(q, 1),(r, 1)^{2},(q, 1)\right]^{k},(r, 1),(q, 1),(p, 1),(r, 1)^{2},(p, 1),\left[(p, 1),(r, 1)^{2},(p, 1)\right]^{k},(q, 1)^{n}\right)$ if $n=2 k+2$.

Proposition 5.1 For all $n$ in $\mathbb{N}^{*}$, the root scheme $R S_{n}$ is realizable by polynomials of degree $2 n$.

Proof. According to lemma 4.5. one can substitute $(p, 1)^{n}$ instead of $(p, n)$ in $R S_{n}$, and according to proposition 4.4 one has just to construct a rational graph on $\mathbb{C} P^{1}$ with a real part corresponding to the real graph of $R S_{n}$. We will prove it by induction on $n$. All the pictures will represent the half $\{\operatorname{Im}(z) \leq 0\}$ of $\mathbb{C} P^{1}$.

The rational graph corresponding to $R S_{1}$ is depicted on Figure 8 ).

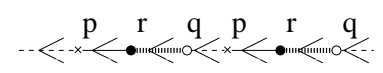

a)

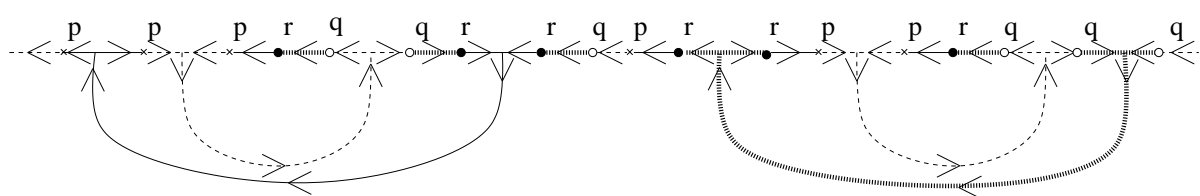

b)

Figure 8:

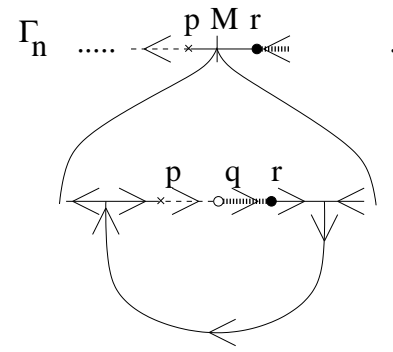

a)

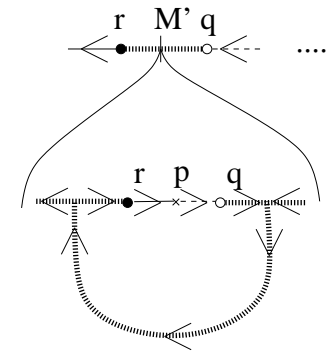

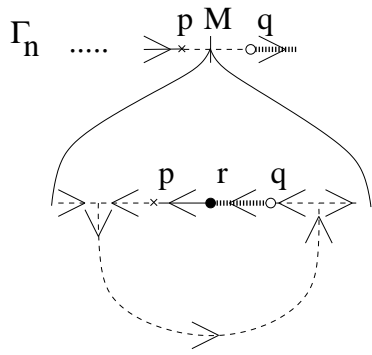

b)

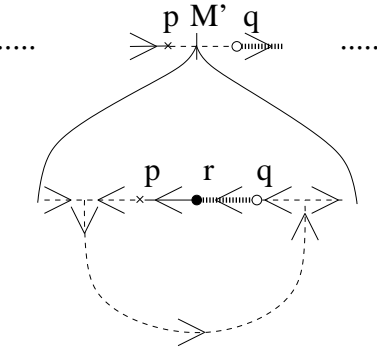

Figure 9:

Suppose that a rational graph $\Gamma_{n}$ corresponding to $R S_{n}$ is constructed. 
Consider, first, the case $n=2 k+1$.

Let $M$ be a point on $\Gamma_{n} \cap \mathbb{R} P^{1}$ between the most right point corresponding to $p$ in $R S_{n}$ and the most left point corresponding to $r$ in $R S_{n}$. Then cut $\Gamma_{n}$ at $M$ and glue the piece of graph depicted in Figure 9a).

Let $M^{\prime}$ be a point on $\Gamma_{n} \cap \mathbb{R} P^{1}$ between the more right point corresponding to $r$ in $R S_{n}$ and the more left point corresponding to $q$ in $R S_{n}$. Then cut $\Gamma_{n}$ at $M^{\prime}$ and glue the piece of graph depicted in Figure 9a).

Consider now the case $n=2 k$.

Let $M$ be a point on $\Gamma_{n} \cap \mathbb{R} P^{1}$ between the more right point corresponding to $p$ in $R S_{n}$ and the more left point corresponding to $q$ in $R S_{n}$. Then cut $\Gamma_{n}$ at $M$ and glue the piece of graph depicted in Figure 9p).

Let $M^{\prime}$ be a point on $\Gamma_{n} \cap \mathbb{R} P^{1}$ between the more right point corresponding to $p$ in $R S_{n}$ and the more left point corresponding to $q$ in $R S_{n}$. Then cut $\Gamma_{n}$ at $M^{\prime}$ and glue the piece of graph depicted in Figure 9b).

For example, $\Gamma_{3}$ is depicted in Figure $8 \mathrm{~b}$ ). According to proposition 4.4 the rational graphs $\Gamma_{n}$ ensure the realizability of the root schemes $R S_{n}$ by polynomials of degree $2 n$.

Corollary 5.2 For all $n$ in $\mathbb{N}^{*}$, there exists three real polynomials $a_{1}(X), a_{2}(X)$ and $b(X)$ of degree $n$ such that

- all the roots of $a_{1}, a_{2}, b$ and $a_{1} b+a 2$ are real,

- all the roots of $a_{2}$ and $a_{1} b+a 2$ are smaller than the roots of $b$.

Proof. Let $P(X), Q(X)$ and $R(X)=P(X)+Q(X)$ three polynomials of degree $2 n$ realizing the root scheme $R S_{n}$. Then

- $Q(X)=\prod_{i=1}^{2 n}\left(X-y_{i}\right)$ with $y_{1}<y_{2}<\ldots<y_{2 n}$,

- $P(X)=(X-\alpha)^{n} \prod_{i=1}^{n}\left(X-x_{i}\right)$ with $\alpha<x_{1}<x_{2}<\ldots<x_{n}<y_{n+1}$,

- $R(X)=\prod_{i=1}^{2 n}\left(X-z_{i}\right)$ with $z_{1}<z_{2}<\ldots<z_{2 n}<y_{n+1}$.

Let us define $a_{2}(X)=(X+\alpha)^{2 n} P\left(-\frac{1}{X+\alpha}\right), A_{1}(X)=\prod_{i=1}^{n}\left(X-y_{i}\right), a_{1}=(X+\alpha)^{n} A_{1}\left(-\frac{1}{X+\alpha}\right)$, $B(X)=\prod_{i=n+1}^{2 n}\left(X-y_{i}\right)$ and $b=(X+\alpha)^{n} B\left(-\frac{1}{X+\alpha}\right)$.

As $a_{1} b+a 2=(X+\alpha)^{2 n} R\left(-\frac{1}{X+\alpha}\right)$, the corollary follows from the definition of $P, Q$ and $R$.

Now we are able to prove proposition 3.1

Proof of proposition 3.1. We construct here explicitly only curves in $\Sigma_{2 k}$. The construction of curves in $\Sigma_{2 k+1}$ is done in the same way. Let us fix an even $n \geq 1$ and consider the polynomials $a_{1}(X)$, $a_{2}(X)$, and $b(X)$ of degree $n$ constructed in corollary [5.2. Multiplying these three polynomials by -1 and performing a linear change of coordinates if necessary, we can assume that the leading coefficient of $b$ is positive, all the roots of $b$ are positive, and all the roots of $a_{2}$ and $a_{1} b+a_{2}$ are negative. Then, the curve $Y(Y-b(X))$ in $\Sigma_{n}$ is depicted in Figure 10]).

For $t$ small enough and of suitable sign, the relative positions of the curves $D_{n}(X, Y)=Y(Y-$ $b(X))+t\left(a_{1}(X) Y+a_{2}(X)\right),\{Y=0\}$ and $\{Y-b(X)=0\}$ are as depicted in Figure 10b), where $p$ is a tangency point of order $n$ of $D_{n}$ and the axis $\{Y=0\}$. Indeed, the definition of $a_{1}(X)$, 


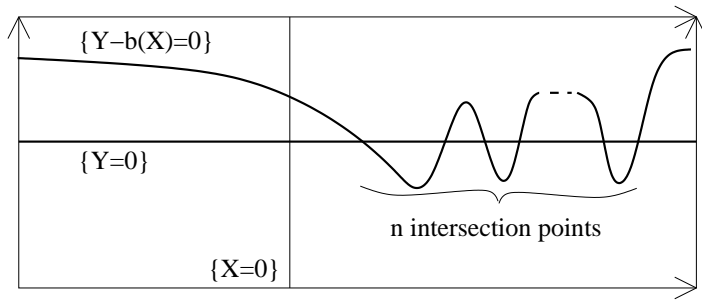

a)

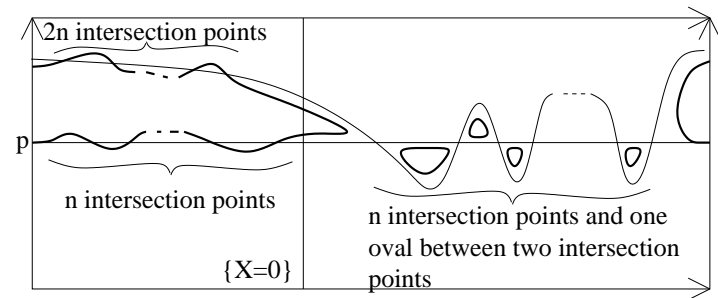

b)

Figure 10:

$a_{2}(X)$, and $b(X)$ exactly means that the intersection points of $a_{1}(X) Y+a_{2}(X)$ and $Y(Y-b(X))$ have negative abscissa. Perturbing all the double points of $D_{n}(X, Y)(Y-b(X))$ in order to have the maximal number of ovals and keeping the tangency point of order $n$ with the axis $\{Y=0\}$, we obtain a curve $C_{n}$ with $n$ even, satisfying the conditions of proposition 3.1

\section{Applications to real algebraic surfaces}

Here we recall and follow the notations proposed in Bih. We consider only $\mathbb{Z} / 2 \mathbb{Z}$-homology. Let $d, i, k$ and $n$ be integers, $\beta_{i}\left(\mathbb{R} X_{d}^{n}\right)$ the $i^{\text {th }}$ Betti number of the real part of a nonsingular real hypersurface of degree $d$ in $\mathbb{C} P^{n}$, and $\beta_{i}\left(\mathbb{R} Y_{2 k}^{n}\right)$ the $i^{t h}$ Betti number of the real part (for some real structure) of a double covering of $\mathbb{C} P^{n}$ branched along a nonsingular real hypersurface of degree $2 k$. An interesting question concerns the asymptotic behavior of $\max \beta_{i}\left(\mathbb{R} X_{d}^{n}\right)$ and $\max \beta_{i}\left(\mathbb{R} Y_{2 k}^{n}\right)$ when $d$ and $k$ go to infinity. In Bih, Bihan has showed that there exist two sequences of real numbers $\left(\zeta_{i, n}\right)_{i, n \in \mathbb{N}^{2}}$ and $\left(\delta_{i, n}\right)_{i, n \in \mathbb{N}^{2}}$ such that

$$
\max \beta_{i}\left(\mathbb{R} X_{d}^{n}\right) \underset{d \rightarrow \infty}{\sim} \zeta_{i, n} d^{n} \quad \text { and } \quad \max \beta_{i}\left(\mathbb{R} Y_{2 k}^{n}\right) \underset{k \rightarrow \infty}{\sim} \delta_{i, n} k^{n} .
$$

The exact value of the numbers $\zeta_{i, n}$ and $\delta_{i, n}$ are known only for small $n$. The following equalities are well known (see [Bih], [te01]).

$$
\begin{gathered}
\delta_{0,0}=2, \zeta_{0,1}=\delta_{0,1}=\delta_{1,1}=1, \zeta_{0,2}=\zeta_{1,2}=\frac{1}{2} \\
\delta_{0,2} \leq \frac{7}{4}, \delta_{1,2} \leq \frac{7}{2}, \quad \zeta_{0,3} \leq \frac{5}{12} \text { and } \zeta_{1,3} \leq \frac{5}{6}
\end{gathered}
$$

The upper bounds are classical and are obtained using the Harnack and Comessati-PetrovskyOleinik inequalities. Lower bounds for $\delta_{0,2}$ and $\delta_{1,2}$ are directly related to the asymptotically maximal number of even ovals of a curve of even degree in $\mathbb{R} P^{2}$, and before the results of the present paper, the best known lower bounds for these two numbers were, respectively, $\frac{27}{16}$ and $\frac{27}{8}$ (see [Ite01]). In Bih, Bihan has constructed nonsingular real algebraic surfaces in $\mathbb{R} P^{3}$ with Betti numbers related to $\delta_{0,2}$ and $\delta_{1,2}$.

Theorem 6.1 (Bihan) One has $\frac{\delta_{0,2}}{6}+\frac{1}{12} \leq \zeta_{0,3}$ and $\frac{\delta_{1,2}}{6}+\frac{1}{6} \leq \zeta_{1,3}$.

Theorem 1.1 gives as immediate corollaries the exact values of $\delta_{0,2}$ and $\delta_{1,2}$ and improves the known lower bounds for $\zeta_{0,3}$ and $\zeta_{1,3}$.

Proposition 6.2 One has $\delta_{0,2}=\frac{7}{4}$ and $\delta_{1,2}=\frac{7}{2}$.

Corollary 6.3 One has $\frac{9}{24} \leq \zeta_{0,3} \leq \frac{5}{12}$ and $\frac{9}{12} \leq \zeta_{1,3} \leq \frac{5}{6}$. 


\section{References}

[Bih] F. Bihan. Asymptotiques de nombres de Betti d'hypersurfaces projectives réelles. Preprint arXiv: math.AG/0312259, (French).

[Bru] E. Brugallé. Symmetric plane curves of degree 7 : pseudo-holomorphic and algebraic classifications. arXiv :math.GT/0404030.

[Haa95] B. Haas. Les multilucarnes: nouveaux contre-exemples à la conjecture de Ragsdale. C. R. Acad. Sci. Paris Sér. I Math., 320(12):1507-1512, 1995. (French).

[IS02] I. Itenberg and E. Shustin. Combinatorial patchworking of real pseudo-holomorphic curves. Turkish J. Math., 26(1):27-51, 2002.

[Ite93] I. Itenberg. Contre-exemples à la conjecture de Ragsdale. C. R. Acad. Sci. Paris Sér. I Math., 317(3):277-282, 1993. (French).

[Ite01] I. Itenberg. On the number of even ovals of a nonsingular curve of even degree in $\mathbb{R} P^{2}$. In Topology, ergodic theory, real algebraic geometry, volume 202 of Amer. Math. Soc. Transl. Ser. 2, pages 121129. Amer. Math. Soc., Providence, RI, 2001.

[NSV02] S. Natanzon, B. Shapiro, and A. Vainshtein. Topological classification of generic real rational functions. J. Knot Theory Ramifications, 11(7):1063-1075, 2002.

[Ore03] S. Yu. Orevkov. Riemann existence theorem and construction of real algebraic curves. Annales de la Faculté des Sciences de Toulouse, 12(4):517-531, 2003.

[Pet33] I. G. Petrovsky. Sur la topologie des courbes réelles algébriques. C. R. Acad. Sci. Paris Sér. I Math., 197:1270-1272, 1933.

[Rag06] V. Ragsdale. On the arrangement of the real branches of plane algebraic curves. Am. J. Math., 28:377-404, 1906.

[San] F. Santos. Unpublished.

[SV03] B. Shapiro and A. Vainshtein. Counting real rational functions with all real critical values. Mosc. Math. J., 3(2):647-659, 745, 2003.

[Vir] O. Ya. Viro. Patchworking real algebraic varieties. http://www.math.uu.se/ oleg.

[Vir84] O. Ya. Viro. Gluing of plane real algebraic curves and constructions of curves of degrees 6 and 7. In Topology (Leningrad, 1982), volume 1060 of Lecture Notes in Math., pages 187-200. Springer, Berlin, 1984.

[Vir89] O. Ya. Viro. Real plane algebraic curves: constructions with controlled topology. Leningrad Math. J., 1(5):1059-1134, 1989.

[Zvo] A. Zvonkin. Unpublished.

\section{Erwan Brugallé}

Laboratoire Emile Picard

Université Paul Sabatier

UFR MIG

118 route de Narbonne

31000 Toulouse

FRANCE

E-mail : brugalle@picard.ups-tlse.fr 